The last two papers were on metal carbonyls. Frequently to-day, new preparative methods involve the use of reagents that a fow years ago were considered too difficult or too dangerous to handle because of the risks of explosion, fire or toxicity. The hazards of using acetylene under pressure and the toxicity of carbonyls frequently cause these reagents to be avoided and it is hoped that these pepers, in which safe methods of handling were discussed by the authors, may alleviate some of these fears.

Dr. Whiting stressed the usefulness of nickel carbonyl in reacting with acetylenes having a terminal triple bond to give $\alpha$-substituted acrylic acids, a type of compound otherwise rather inaccessible. These resctions are carried out in acetic acid at moderate temperature, with excess carbonyl which is not difficult to handle provided that its transfer from vessel to vessel is carried out under conditions involving only a very slight degree of exposure to air, and provided that excess is removed from the reaction mixture by co-distillation with ether. The distillate is immediately disposed of and leaves a reaction mixture that can safely be handled in the usual way. Even so, both he and Dr. Lenel pointed out that although these substances could be handled safely they still had to be treated with considerable respect. Dr. Whiting also went at some length into the mechanisms of these reactions. He was followed by Dr. Lenel, who dealt mostly with the industrial applications of carbonyls in reaction with acetylenes to give unsaturated acids, or with olefines to give saturated acids. Cobalt carbonyl catalyses the important reaction of carbon monoxide and hydrogen with olefines to give aldehydes which can then be reduced to alcohols by hydrogenation. The industrial preparation of alcohols and acids by these reactions is now carried out on a very large scale involving many thousands of tons of product per annum, though the reactions necessitate fairly high temperatures and pressures. Several other carbonyls of transitional elements are known, though they have not yet achieved prominence in an industrial sense; they are of profound interest to the inorganic and theoretical chemist because their constitutions throw new light on electronic structures.

This was, as Prof. Hirst pointed out, the first joint meeting of the Chemical Society and the Fine Chemicals Group of the Society of Chemical Industry, and it is very much to be hoped that it will be followed by others. The members of the Fine Chemicals Group are predominantly interested in the pre. paration of complex chemicals in a high degree of purity : perfumes, photographic sensitizers, drugs, vitamins and hormones, for example. These processes often involve twelve to twenty stages that, if they are to be carried out efficiently, demand the use of the most up-to-date knowledge and techniques available. This Group, of all the special groups in the Society of Chemical Industry, is the one that has the strongest kinship with the Chemical Society. With the rapidly increasing activity in industrial chemistry of all kinds and the rapid extension of industrial technical staffs, joint meetings of this kind can be a valuable aid to progress. A closer relationship between the members of the two Societies can be of great benefit to both.

\title{
INTERACTION IN IONIC SOLUTIONS
}

$\mathrm{T}$ HE Faraday Society held a general discussion on "Interaction in Ionic Solutions" at Oxford during September 17-19. The meeting was held in the University Laboratory of Physiology, and more than 180 members and visitors stayed in Balliol College, where the guest-night dinner was held. The president of the Society, Mr. R. P. Bell, in opening the discussion, reminded those present that a general discussion on "Electrolyte Solutions" had been held thirty years ago in Oxford. In fact, the president's first published contribution to the subject was presented at that meeting in 1927 in a joint paper with Sir Harold Hartley. Among others present, who had attended and contributed to the earlier discussion, was Prof. H. S. Harned (Yale), who delivered the Spiers Memorial Lecture on "Some Recent Experimental Studies of Diffusion in Liquid Systems". Prof. Harned gave an account of the elegant experimental techniques for the precision measurement of diffusion in electrolytes which have been recently developed at Yale, and indicated some of the many possible directions of further advance. The accurate determination of activity coefficients by diffusion measurements is a remarkable achievement.

The first paper to be discussed was that by Prof. H. Falkenhagen and Dr. G. Kelbg (Rostock), which dealt with the extension of the limiting-law equations. Dr. M. Eigen (Göttingen) directed attention to the information that can be obtained about the intimate details of short-range interaction in bi-bivalent electrolytes by the observations of relaxation effects in sound-absorption spectra. Dr. S. Levine and Mr. H. E.
Wrigley (Manchester) reported a calculation of the interaction of two univalent ions at small separations, and considered the relevance of the macroscopic dielectric constant of the solvent in this situation. In discussion, Prof. L. Onsager (Yale) directed attention to some recent mathematical advances which might help in the electrolyte problem, while Prof. H. S. Frank (Pittsburgh) seized the opportunity to express heretical views about the Debye-Hückel theory. A paper by Prof. T. F. Young, Mr. Y. C. Wu and Dr. A. A. Krawetz (Chicago) reported some measurements of the heat effects due to interactions between ions of like charge when pairs of univalent electrolytes with an anion or cation in common are mixed.

There followed a major section devoted to the presentation and discussion of papers dealing with the incomplete-dissociation model as a way of handling short-range ion-ion interactions. Prof. C. W. Davies (Aberystwyth) surveyed the present position in an introductory paper. Prof. E. A. Guggenheim (Reading) considered the ion distribution in dilute aquoous solutions of single binary electrolytes, and concluded that although the Debye-Hückel approximation is invalid for bi-bivalent sulphates in water, the model (complete ionization to rigid spherical ions) is satisfactory if, following $N$. Bjerrum, ions of opposite charge approaching more closely than 10-14 A. are treated as associated. Dr. O. Redlich and Dr. G. C. Hood (Shell Development Co., California) presented the results of Reman and nuclear magnetic-resonance investigations of the dissociation of strong acids (including nitric, perchloric and sul- 
phuric acids). The agreement between the results is very satisfactory. Mr. W. D. Bale, Mr. E. W. Davies, Mr. D. B. Morgans and Dr. C. B. Monk (Aberystwyth) reported on the study of various ion association equilibria by spectrophotometry. Mr. W. G. Davies, Mr. R. J. Otter and Dr. J. E. Prue (Reeding) reported precise spectrophotometric results for copper sulphate solutions, and discussed the significance of the dissociation constants obtained from cryoscopic, conductimetric and spectrophotometric measurements. Dr. G. H. Nancollas (Glasgow) gave an account of attempts to obtain and interpret heat and entropy changes for ion association reactions. In the discussion of this section there was general agreement about the validity and usefulness of the incomplete dissociation model for higher-valent electrolytes, but there was some dissension, particularly between Prof. Davies and Dr. Prue, about the relation of the numerical values of the dissociation constants to the choice of a distance of closest approach of free ions.

A section on ion-solvent interaction was introduced by Prof. H. S. Frank and Dr. Wen-Yang Wen (Pittsburgh), who discussed the several ways in which they consider ions affect the structure of water. Dr. O. T. Samoilov (Moscow) had submitted a paper on "A New Approach to the Study of Hydration of Ions", but was unfortunately absent. Dr. S. R. Gupta, Dr. G. J. Hills (Imperial College) and Dr. D. J. G. Ives (Birkbeck College) considered that their recent very precise measurements of activity coefficients of aqueous hydrochloric acid solutions over a temperature-range provide evidence for changes in heat capacity which can be interpreted in terms of changing ion-solvent interactions. A theoretical paper by Dr. A. D. Buckingham (Oxford) led to the conclusion that the ion-quadrupole term is significant in the interaction between the ion and solvent molecule, and that the dielectric-saturation effect is unimportant outside the first hydration sphere.
Few real advances or firm conclusions were discernible in the ion-solvent interaction section. However, there is considerable evidence for the existence of the proton as a tetrahydrate in water. It is entirely reasonable that a hydroxonium ion forms strong hydrogen bonds with three neighbouring water molecules and Dr. K. N. Bascombe and Mr. R. P. Bell (Oxford) showed that the variation of Hammett acidity functions with concentration in concentrated strong acids can be satisfactorily interpreted with such a model. Their general conclusion was supported by Dr. Th. Ackermann's (Hamburg) study of the heat capacity and infra-red absorption spectra of aqueous acid and alksli solutions. Further support was provided by the X-ray diffraction studies of aqueous electrolyte solutions by Mr. C. L. van Panthaleon van Eck, Mr. H. Mendel and Mr. W. Boog (Royal Dutch Shell, Amsterdam). This method shows considerable promise as a direct way of exploring the solvation problem; present results suggest that water molecules in water and potassium and calcium ions are octahedrally surrounded by six water molecules.

Dr. P. A. H. Wyatt (Sheffield) considered the interpretation of acidity functions in concentrated acids. Dr. M. Kaminsky (Marburg) discussed the relationship between the dependence on temperature of viscosity of aqueous solutions and the ion-solvent interaction. A paper on the dependence on concentration of the surface potentials of a number of electrolyte solutions by Dr. J. E. B. Randles (Birmingham) suggested that the interpretation of the results is too complex to lead to reliable evidence about ion hydration. Mr. M. Smith and Dr. M. C. R. Symons (Southampton) presented a study and interpretation of the effects of changes of solvent, added electrolyte and temperature on the first electronic absorption band of the iodide ion.

J. E. Prue

\section{REORGANIZATION OF THE METEOROLOGICAL OFFICE}

$I^{N}$ N 1955, the Secretary of State for Air appointed a Committee under Lord Brabazon to review the organization of the Meteorological Office. This Conumittee took evidence from users, both inside and outside Government service, of the services provided by the Meteorological Office, and from members of the Office staff. The main features of the Committee's report, which will not be published, were given to the House of Commons in June by the Minister for Air.

The Committee agreed that the Air Ministry should continue to be responsible for the Meteorological Office, and expressed the opinions that the standing of the Office as a scientific institution is high and that users have considerable confidence in the services provided for them. In considering possible lines of advance the Committee singled out the development of numerical methods of forecasting as one of the most important, welcomed the forthcoming installation of an electronic computer in the Office, and suggested there was a possibility of achieving greater precision in local forecasting by a closer study of weather characteristics and by the use of the radar-echo method of observing cloud and precipitation.

As regards internal arrangement, the Committee recommended that the Office should be reorganized into three"'prongs' dealing, respectively, with forecasting and services, research, and administration. This reorganization was put into force in July 1957. Its recommendations also led to a regrading of the senior pusts whereby the post of Director has been raised to that of Director-General, the forecasting and services 'prong' is placed under the Director of Services and research under the Director of Research, each of the two latter posts being in the grade of chief scientific officer. 'The administrative 'prong' now under an assistant secretary has been enlarged to make the Office more self-contained in administrative matters.

Several new posts in the grade of senior principal scientific officer have also been created, and the number of the highest pusts available to members of the experimental officer class will also be increased. These measures will improve the career prospects, a matter on which the Brabazon Committee expressed considerable concern, for all classes of the staff. The need for improvement was especially evident in the scientific officer class, to which it had been difficult to attract recruits of sufficiently high ability.

On the Services side the new posts include a senior principal scientific officer to control 'Techniques and 